\title{
Paper II
}

G. Yang, J. Chen, L. Xie, J. Mao, T. Hannu, and L.-R. Zheng “A Hybrid Low Power BioPatch for Body Surface Potential Measurement” IEEE Journal of Biomedical and Health Informatics (J-BHI), 2013 (accept). 


\title{
A Hybrid Low Power Bio-Patch for Body Surface Potential Measurement
}

\author{
Geng Yang, Jian Chen, Li Xie, Jia Mao, Hannu Tenhunen, and Li-Rong Zheng
}

\begin{abstract}
This paper presents a wearable Bio-Patch prototype for body surface potential measurement. It combines three key technologies, including mixed-signal System on Chip (SoC) technology, inkjet printing technology and anisotropic conductive adhesive (ACA) bonding technology. An integral part of the Bio-Patch is a low-power low-noise SoC. The SoC contains a tunable analog front end, a successive approximation register analog to digital converter, and a reconfigurable digital controller. The electrodes, interconnections and interposer are implemented by inkjet-printing the silver ink precisely on a flexible substrate. The reliability of printed traces is evaluated by static bending tests. ACA is used to attach the SoC to the printed structures and form the flexible hybrid system. The Bio-Patch prototype is light and thin with a physical size of $16 \times 16 \mathrm{~cm}^{2}$. Measurement results show that low-noise concurrent electrocardiogram signals from 8 chest points have been successfully recorded using the implemented Bio-Patch.
\end{abstract}

Index Terms-body surface potential, Active Cable, inkjet printing, ACA, wearable device, SoC, Bio-Patch.

\section{INTRODUCTION}

$\mathrm{W}$ ITH the developments in complementary metal-oxide semiconductor (CMOS) technology, fully integrated design has shown advantages in many advanced medical applications, in terms of low-power consumption and high-level integration. Examples can be found in battery-powered portable, wearable or even implantable medical devices [1] [2]. One of the current trends in healthcare systems is to move parts of healthcare services, such as routine check for senior citizens, from hospitals to home environments [3]. Thus release the limited hospital resources to the people in emergency needs. Home healthcare can not only lighten the workload of doctors and nurses but also allow people to self-monitor their health condition anytime. Thus patients can become actively involved

Manuscript received June 15, 2012. This work was financially supported by Vinnova (The Swedish Governmental Agency for Innovation Systems) through the Vinn Excellence centers program.

Geng Yang, Jian Chen, Li Xie, Jia Mao and Hannu Tenhunen are with iPack Vinn Excellence Center, School of Information and Communication Technology, Royal Institute of Technology (KTH), Forum 120, 164-40 Kista-Stockholm, Sweden (e-mail: gengy@kth.se).

Li-Rong Zheng is with iPack VINN Excellence Center, School of Information and Communication Technology, the Royal Institute of Technology (KTH), SE 164-40 Kista-Stockholm, Sweden, and also with the State Key Laboratory of ASICs and Systems, Fudan University, 200433, Shanghai, China (e-mail: lirong@kth.se)

Copyright (c) 2007 IEEE. Personal use of this material is permitted. in their own care process [3]. On the other hand, the technology trends for wearable medical devices are to develop unobtrusive, miniaturized, integrated, networked, and long lasting medical sensor devices [3] [4].

Heart monitoring is an essential part in home healthcare, since heart disease is chronic process and is one of the most common causes for death all over the world [5]. Body surface potential measurement has proven to be a clinically relevant method that enhances diagnostic ability due to the larger number of sampling electrode positions on the thorax [6]. It can be obtained by processing the concurrently recorded multi-channel electrocardiogram (ECG) signals [6]. However, traditional ECG recording technology suffers from the massive connecting cables between the electrodes and the processing equipment. Examples can be found in a standard 12-lead ECG monitoring, where 10 recording electrodes are used. Additional electrodes may also be added to the standard set-up when increased spatial resolution is needed. This may cause to the following problems: 1 ) it is uncomfortable and inconvenient for the users if wires are worn around the body all day long; 2) the wires can easily get tangled; detangling is time consuming; 3 ) electrodes placement and configuration require professional assistance from nurses or healthcare professionals; any faulty connection may lead to a misdiagnosis. The facts above become limiting factors when considering the wider use of medical devices in home healthcare applications.

Based on these considerations, we develop a low power Bio-Patch for wearable body surface potential measurement. It is implemented by integrating a customized System on Chip (SoC) on a flexible polyimide (PI) substrate. The system is capable of concurrently detecting ECG signals from 8 different points across the heart. Conductive electrodes are assembled beneath the patch, which eliminate the connecting cables and avoid electrodes mis-configuration problem.

As a key part, a mixed-signal SoC with a full integration of processing modules is employed to fulfill the signal acquisition and processing. The advantages of using a SoC include significant power saving compared with its counterparts using off-the-shelf components. Also from physical dimension point of view, sensor size is a key factor which directly affects user's comfort. Some research groups make their efforts to develop sensor node using off-the-shelf components, while the resulting size is rather big [7, 8], since too many discrete components are employed, as a result, they are not suitable for wearable healthcare devices. Sensor device miniaturization can be realized by applying this compact $\mathrm{SoC}$ in the Bio-Patch implementation, since fewer external components are required. 


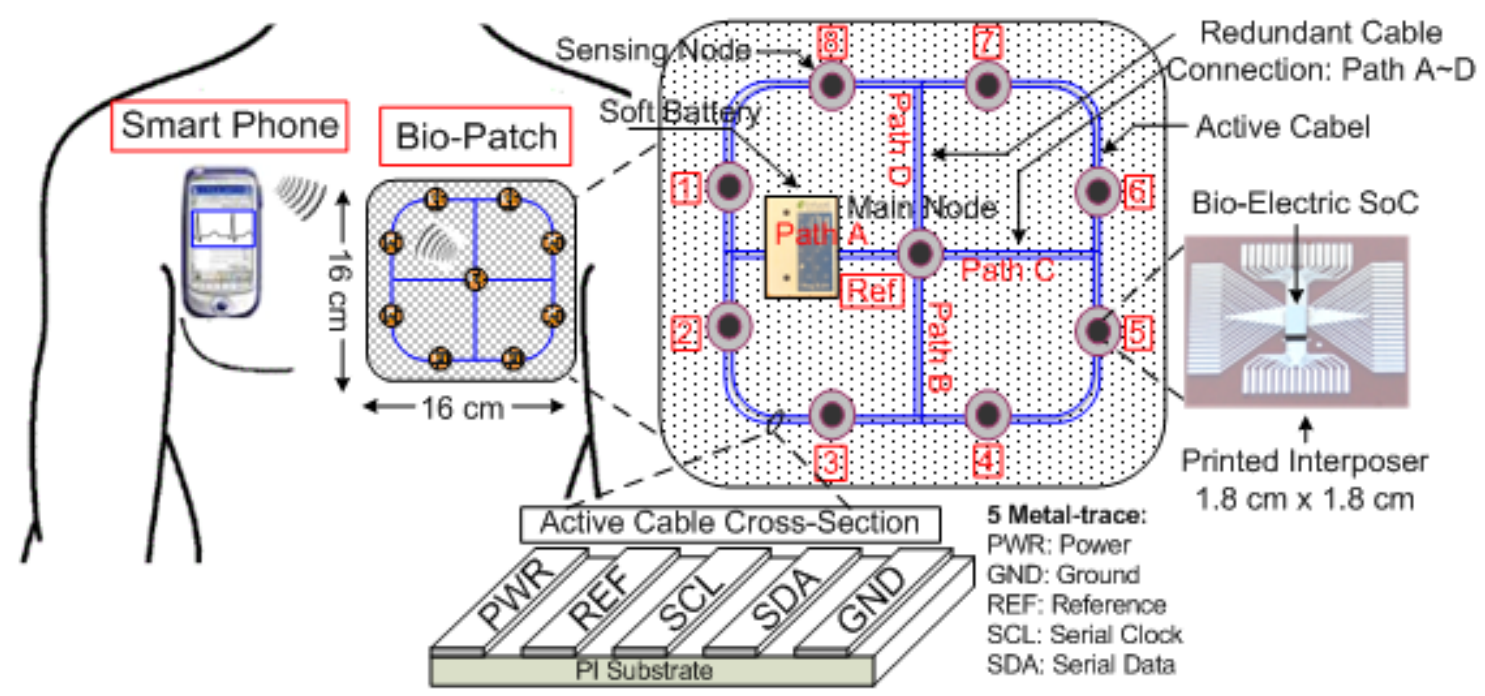

Fig. 1. Overall architecture of the proposed wearable Bio-Patch.

A serial communication protocol is implemented on chip. The output from each sensor node is a serial data stream which is transmitted from node to node along a 2-wire serial link. The integrated digital core enables the SoC to set up a network using a variety of unicast or broadcast commands. In addition, the noise resistance of the detecting circuit and power consumption of the whole system are the major considerations in the SoC design. To effectively reject the environmental noise, a sensing circuit with a common-mode rejection ratio (CMRR) of $90 \mathrm{~dB}$ is designed by employing a fully differential current balancing architecture. The total power consumption of the SoC is $20 \mu \mathrm{W}$ from a $1.2 \mathrm{~V}$ battery.

PI substrate is selected as the patch carrier, since it is low-cost, flexible (wearable), printable, thin and light [9]. High resolution inkjet printing is utilized to fabricate the printed devices [10]. Advantages of using this additive manufacture process include: less process steps, lower cost and better environmental compatibility, since inkjet printing technology can avoid the masks preparation and etching steps. Therefore, it is suitable for customized bio-sensing electrodes, lead frames as well as other patterns according to specific requirements [11]. A printed Bio-Patch is formed by assembling the inkjet printed interposer lead-frame, conductive electrodes, and printed circuit board on the PI substrate. Anisotropic conductive adhesive (ACA) is an environmentally friendly technique, which has been widely used in flip chip bonding applications with a wide range of substrate [12]. In this design, ACA is employed to bond the Bio-Electric SoC chip onto the PI interposer to form a sensor node. A soft battery from Enfucell is attached to the Bio-Patch as power supply.

The proposed hybrid wearable body surface potential acquisition system integrates silicon based electronics with printed electronics, and takes advantages of both [13]. The rest parts of the paper are organized as follows: system architecture is presented in Section II; SoC design and implementation are presented in Section III. Bio-Patch prototype assembly process and in-vivo tests are shown in Section IV. Finally, discussion and conclusion are made in Section V.

\section{SYSTEM ARCHITECTURE}

The proposed wearable Bio-Patch is shown in Fig. 1. It is attached on the subject's chest over the heart area, and can concurrently detect 8 points ECG signals. The patch is implemented on a flexible PI substrate, and composed of 9 sensor nodes. For each sensor node, a SoC is embedded inside. Due to the reconfigurable architecture of the digital core, each sensor node has two operation modes: the one located in the middle of the patch is configured as a Main-Node (MN), while the other 8 sensor nodes placed around are configured as Sensing-Nodes (SNs), each SN represents one sensing channel, as shown in Fig. 1. The MN manages the whole system, while the SN performs ECG signal acquisition. The detected ECG signal is stored locally in a SN's on-chip RAM before periodically collected by the MN.

A two-wire serial transmission protocol is implemented on-chip. It enables the serial communication between different sensor nodes. Therefore, a body area network is established over a group of sensor nodes. The output from each $\mathrm{SN}$ is serial data stream which is periodically retrieved by the MN and finally sent to a smart phone or a base station through MN's wireless interface. Commercially available wireless transmission module, such as Bluetooth, can be employed for wireless transmission. While a custom designed wireless transmitter, a novel all-digital polar transmitter, is also investigated for this design. It is implemented and tested on another chip, the measurement results are reported in [14].

An Active Cable [15] [16] is proposed to serially connect each sensor node to form a wired network. Its cross-section view is illustrated in Fig. 1. It is composed of five silver traces (PWR, GND, REF, SCL and SDA) with the thickness of $0.5 \mu \mathrm{m}$ by printing the silver ink on the PI substrate (thickness of 30 $\mu \mathrm{m})$. The Active Cable serves two purposes: 1) to share the power (PWR), ground (GND) and reference signal (REF); 2) to establish a wired network via a serial link (SCL and SDA). Comparing with the traditional star-topology full cable solution, 


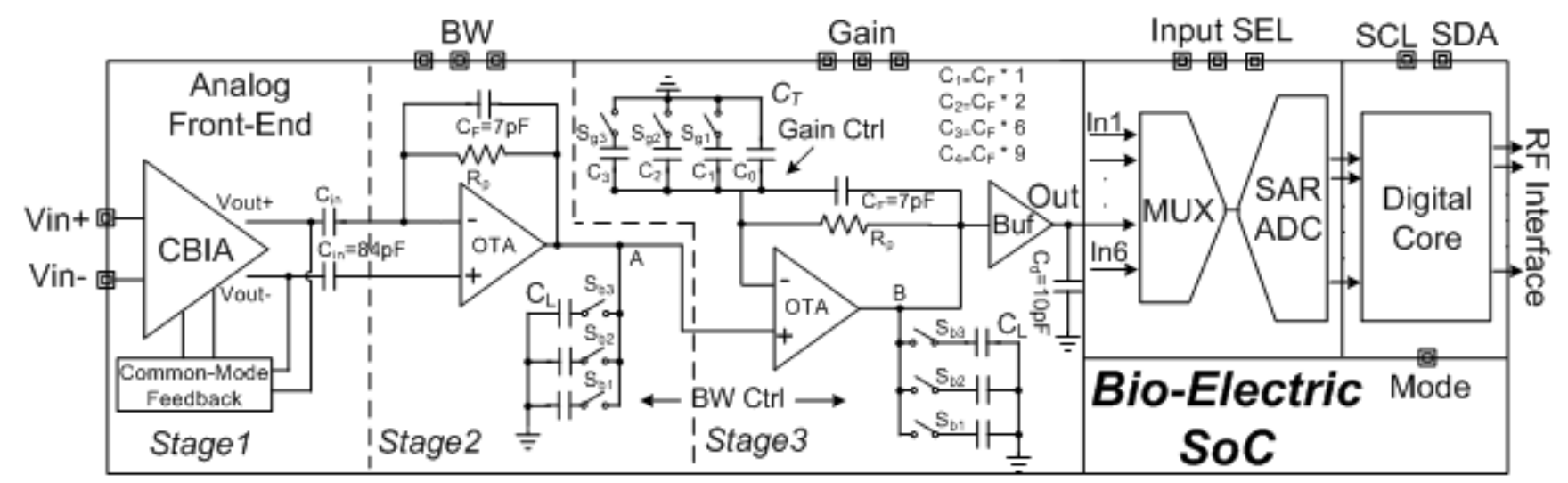

Fig. 2. Block diagram of the Bio-Electric SoC.

this serial connection method significantly reduces the number of connecting cables by sharing the signal chain among multiple sensor nodes, and hence improves users' comfort.

A typical ECG signal extraction flow can be described as follows: the $\mathrm{MN}$ provides its local electrical potential as a system reference (REF in the Active Cable). This REF signal is spread through out the whole patch to each SN via the Active Cable. While for a SN, REF is connected to one input of the AFE; a local voltage (detected from the electrode attached beneath the $\mathrm{SN}$ ) is connected to the other input. The differential sensing is performed by subtracting the REF voltage from the time-varying local voltage. The extracted bio-signal is amplified and filtered by the front end circuit, and digitized by the on-chip analog to digital convertor (ADC). The digital core buffers the digital data temporarily in one on-chip RAM and transmits them out via the serial bus before the memory is full.

A common concern encountered by almost all the inkjet printed circuits on flexible substrate is the cracks in the printed lines caused by a large substrate bending. In order to ensure the system reliability, Active Cables employs a redundant architecture (Path A - Path D in Fig. 1) to connect the central MN with other SNs deployed around. Redundant connections allows reliable connectivity in cases where an electric connection is failed in some way. As long as 1 of the 4 redundant cables remains intact, the central controller can find its way to communicate with a specific sensing node, consequently, control the whole network. In order to further investigate the reliability of the printed Active Cable as well as other interconnections printed on the PI substrate, a bending test is performed in Section IV-B. The measurement results indicate that the printed silver traces can provide reliable electric connections, no crack happens even in the extreme case where the substrate is folded in half along the middle line.

\section{BIO-ELECTRIC SOC}

High level integration is accomplished by packing the essential signal processing blocks and control units on the same chip. The implemented Bio-Electric SoC contains three building blocks: 1) a tunable analog front end (AFE) circuit 2) a 6-input 8-bit successive approximation register analog to digital converter (SAR-ADC) and 3) a reconfigurable digital core. The overall architecture of the SoC is illustrated in Fig. 2. The SoC is fabricated in a standard 0.18- $\mu \mathrm{m}$ Mixed-Mode 1P6M CMOS process and totally occupies $1.5 \mathrm{~mm} \times 3 \mathrm{~mm}$ silicon area with a operating voltage ranging from $1.2 \mathrm{~V}$ to $3.3 \mathrm{~V}$. The chip microphotograph is shown in Fig. 3.

\section{A. AEF Readout Circuit and ADC}

The ECG signal amplitude varies from one subject to another. In addition, variations of skin-electrode contact impedance, electrode size, and electrodes distance may also contribute to the fluctuation of the detected ECG amplitude. Therefore, to enable the detection of a wide range of bio-potential signals, a tunable gain is desired for the readout stage. Fig. 2 illustrates the block diagram of the programmable three-stage AFE. It allows us to adjust the gain of the amplification chain to an optimum value, while avoiding saturation distortion. Stage1 provides a fixed gain of 7. It is mainly composed of a current balanced instrument amplifier (CBIA) [17] and a common mode feed back circuit. The differential outputs of Stage1 are AC-coupled to Stage2. The close-loop gain of Stage2 is set to 12. Stage3 provides a tunable gain by tuning the equivalent capacitance of its inner capacitor bank via three external switches. The in-band gain of the Stage3 can be set to eight different values ranging from 2 to 19 . The post-layout simulation results show that the three-stage front-end circuit can provide a tunable close-loop gain from 45 $\mathrm{dB}$ to $64 \mathrm{~dB}$. The low-pass frequency of the front-end can be tuned to $80 \mathrm{~Hz}, 260 \mathrm{~Hz}, 400 \mathrm{~Hz}$ or $1 \mathrm{kHz}$ by varying the load capacitance through the external switches. The high-pass frequency is set to $0.3 \mathrm{~Hz}$. The AFE totally draws a current of $2.3 \mu \mathrm{A}$ from a $1.2 \mathrm{~V}$ supply.

A SAR-ADC is employed due to its relatively good tradeoffs between power efficiency, conversion speed and resolution. A MUX is used to select one from six inputs to connect to the ADC. In this design, one input is reserved for the amplified bio-signal from the AFE; while the other five inputs are available for future sensor node's extension for other bio-signal sensing. The ADC provides a sample rate of $3.3 \mathrm{kS} / \mathrm{s}$, working with an external clock of $100 \mathrm{kHz}$ and consumes $1.8 \mu \mathrm{A}$ from a $1.2 \mathrm{~V}$ supply. 


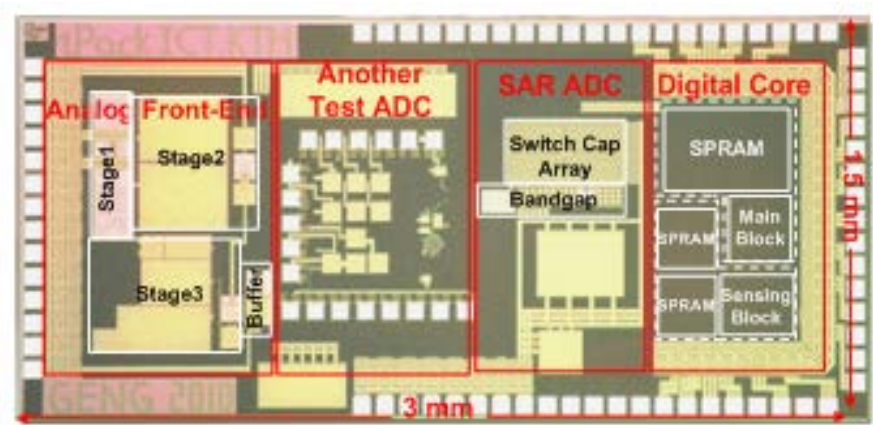

Fig. 3. Chip microphotograph with building blocks marked.

\section{B. AFE Measurement Results}

The gain and bandwidth measurement results of the AFE are plotted in Fig. 4. The measured largest gain (63 dB) is slightly smaller than the simulation result $(64 \mathrm{~dB})$ which may due to the slight attenuation of the unit buffer located at the output port of the AFE. The gain or bandwidth can be independently adjusted via external pins. Totally eight different gain, ranging from 45 $\mathrm{dB}$ to $63 \mathrm{~dB}$, can be selected (three of them chosen for illustration). For each selected gain, four user-programmable 3-dB bandwidths can be obtained using bandwidth switches. The upper part of Fig. 4 shows the case of 3-dB bandwidth adjustment with an almost consistent gain with the low cut-off frequency around $0.3 \mathrm{~Hz}$ and the high cut-off frequency adjusted from $85 \mathrm{~Hz}$ to $850 \mathrm{~Hz}$. Regarding the SoC's electric characteristics, more details have been reported in previous work [18].

Due to its weak amplitude, the bio-signal is prone to be contaminated by the motion noise and other noise sources. In some applications, back-end digital signal processing (DSP) methods are applied to remove the out-of-band noise, however, these approaches consume extra power as well as silicon area. In this work, a fully differential current balancing architecture is applied in the AFE's design to achieve a CMRR of $90 \mathrm{~dB}$, which enables the effective cancellation of the common-mode interference and also relieve the need of DSP. In order to measure input-referred noise (IRN), two input terminals are shorted to circuit ground and the output noise is measured with the signal analyzer (HP Signal Analyzer 35670A). The measured noise divided by the gain at its closet frequency point equals IRN. The IRN floor of $-145 \mathrm{~dB} \mathrm{Vrms} / \sqrt{\mathrm{Hz}}(56 \mathrm{nV} / \sqrt{\mathrm{Hz}})$ is observed.

\section{Digital Core and its Operation}

The on-chip integrated digital core includes three key features: 1) a reconfigurable control logic, which has 2 operation modes; 2) a 2-wire serial transmission protocol, which enables the establishment of a body area network; 3) and three pieces of RAM which allow the sensor node to buffer the digitized bio-signals. The performance of the fabricated digital core has been reported in [19] and [20]. An external pin 'Mode' is used to set the operation mode of each SoC. If the pin is set to logic ' 1 ', the node is configured as a Main-Node (MN); otherwise it is configured as a Sensing-Node (SN). In battery operated systems, great emphasis is put on power saving to extend the

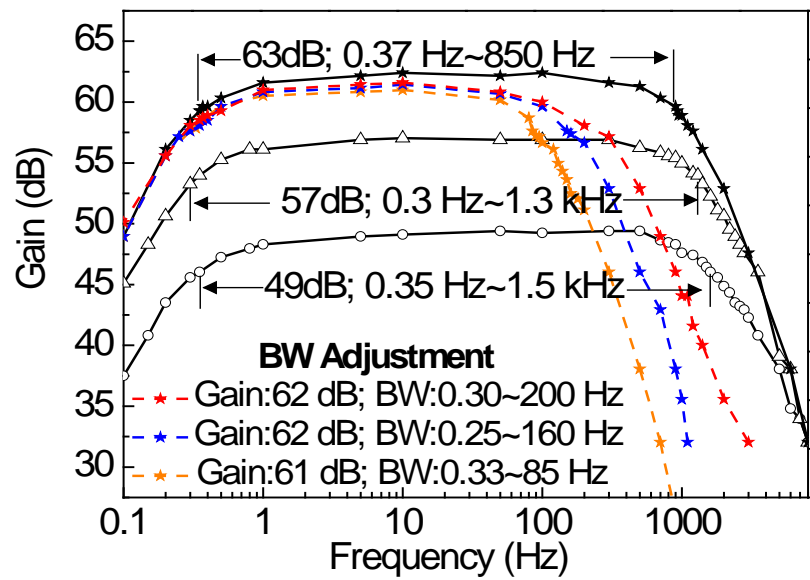

Fig. 4. Gain-bandwidth measurement of the AFE.

battery life. Idle circuit blocks are switched off to minimize the power consumption. For example, the AFE and ADC of the MN is turned off for power saving. Further more, the digital core of the MN spends most of its time in a low-power sleep mode. For the two operating modes, the functionality of the MN and SN differs in:

SN mode: The main function of a $\mathrm{SN}$ is to sample, process and buffer the ECG signal. Each SN has a 4-bit address. The ECG signal from the subject's skin is amplified and digitized. Subsequently, the digital bio-signal is picked up and buffered temporarily in its on-chip RAM. If one RAM is full, the coming data are automatically switched to the second RAM. Before the on chip memory is full, the stored data will be periodically collected by the MN.

$M N$ mode: The main role of the $\mathrm{MN}$ is to manage the whole network using a variety of commands. SN-Chain Scan (SNCS) process is initiated when the system is powered up or reset. During SNCS process, all SNs in the chain are scanned, the address of each active SN is stored in the MN's address buffer. Soon after SNCS is completed, bio-signal data collection process is initiated: the MN visits each $\mathrm{SN}$ in a serial manner. Collected data are buffered in a MN's on-chip RAM. Before the storage is full, the MN forwards these data to an external wireless transmission module, then it continues with the next round data collection.

The SNCS flow is described in Fig. 5a. The MN broadcasts a command packet containing a target SN address to the Active Cable. If the target $\mathrm{SN}$ is available, an acknowledgement frame is sent back immediately. Otherwise, if the target SN does not exist, no acknowledgement will be generated before timer overflow. Subsequently, the MN asserts the absence of the target SN, and continues with the next scan until all SNs are scanned. During this scan process, the ADC located inside in each SN stays in a 'Disabled' state. Fig. $5 \mathrm{~b}$ illustrates the structure of command packet. Fig. 5c shows the screenshot of SCL and SDA on an oscilloscope during SNCS, where the scan of SN ' 1001 ' is enlarged for illustration. For the current version, the system supports one MN and up to 14 SNs. Once SNCS is completed, a synchronous sample ('Syn-Sample') broadcast command is initiated by the MN to trigger the SAR-ADC located in each SN to startup simultaneously. In this way, concurrent sampling of ECG signals from multiple chest 


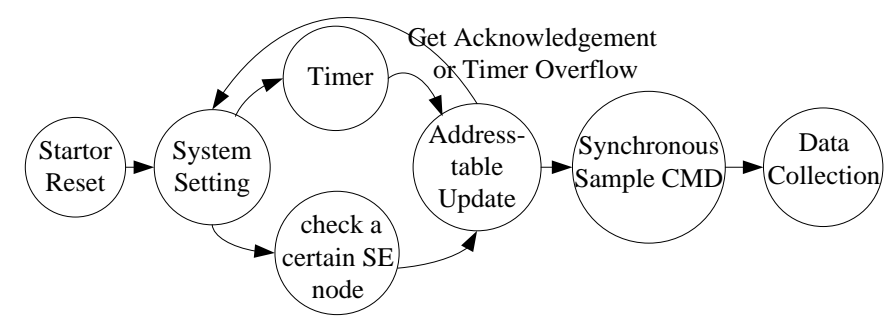

(a)

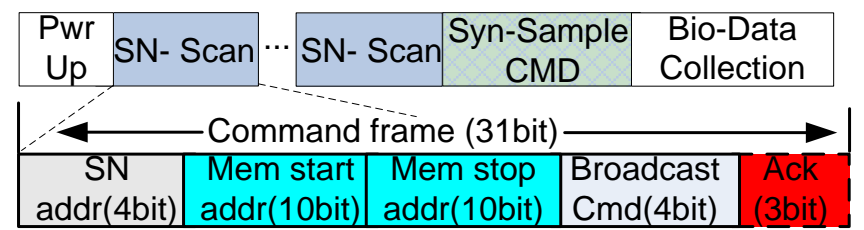

(b)

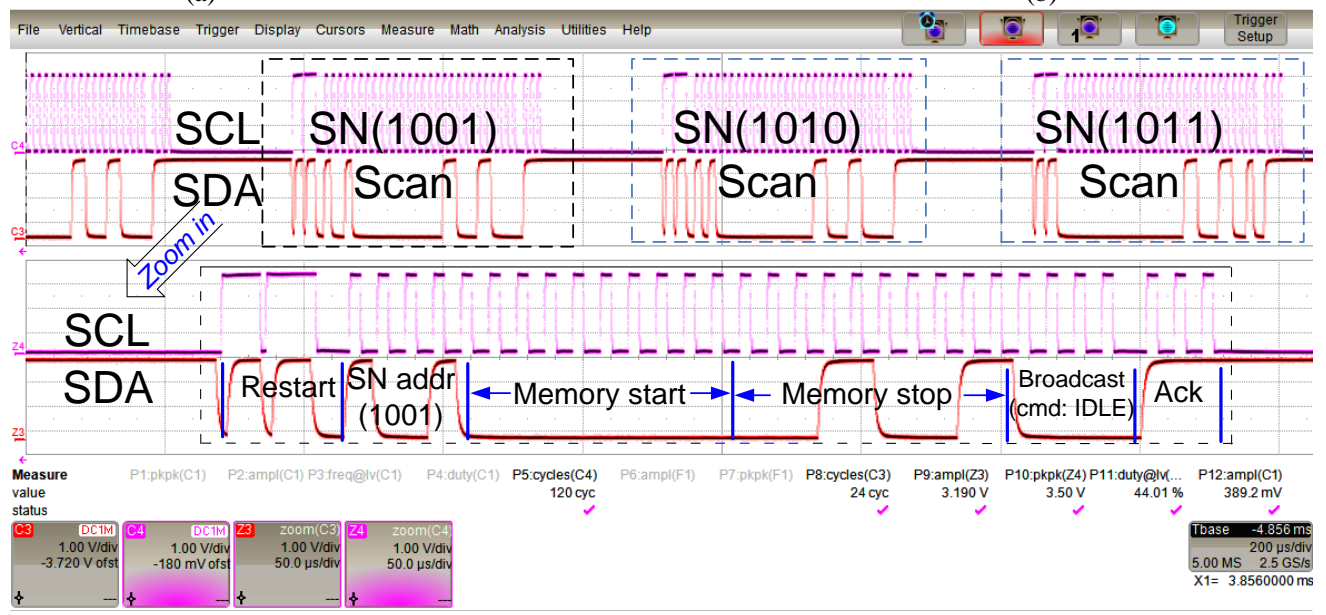

(c)

Fig. 5. (a) Command-based SN-Chain Scan (SNCS) process. (b) Packet structure for the command frame. (c) Oscilloscope screenshot of the SCL and SDA.

positions can be achieved. The average current consumptions of the digital core for the two operation modes are almost the same: $12.7 \mu \mathrm{A}$ for the $\mathrm{MN}$, and $12.6 \mu \mathrm{A}$ for a $\mathrm{SN}$ from $1.2 \mathrm{~V}$ supply with a $1 \mathrm{MHz}$ system clock.

\section{Bio-Patch Assembly Process AND IN-Vivo Test}

\section{A. Assembly Process}

Due to its high resolution and fine spacing, inkjet printing technology is utilized in this work to print conductive silver leads on PI substrate to form an interposer. Silver ink (from Sun Chemical Corp.) is directly printed by inkjet printer (DMP2800 from Dimatix). The volume of each drop is $10 \mathrm{pl}$ and the drop spacing of inkjet processing is set to $20 \mathrm{um}$ (the pad pitch of the SoC is $90 \mathrm{um}$ ). The printed leads exhibit high conductivity (typically around $1.25 \mathrm{E}+7 \mathrm{~S} / \mathrm{m}$ ) and small thickness (around 0.5 $\mu \mathrm{m}$ for one layer printing). The electrical characteristics of the printed silver traces on PI substrate have been investigated and reported in [21]. Fig. 7a represents the printed PI interposer with a dimension of $18 \times 18 \times 0.03 \mathrm{~mm}^{3}$. Cross-marks are made on the lower right corner for chip alignment during assembly. The integration of the tiny-size SoC on the printed interpose makes the sensor node miniaturization possible. The physical size of the interposer is much smaller and thinner than a standard JLCC-86 package $\left(30 \times 30 \times 3 \mathrm{~mm}^{3}\right.$, which was used as the chip container in previous tests). In addition, the JLCC package is not viable for this application due to its large and rigid nature, which makes the user uncomfortable if worn on human body.

ACAs have been widely used in flip chip packaging applications recently [22] [23]. It provides both electrical and mechanical connections between chip pads and the substrate. The distinct advantages of using ACAs include reduced package size and thickness, improved environmental compatibility, and lower assembly temperature [24]. In addition, the process costs are lower compared with traditional bonding/soldering approaches, due to fewer processing steps [25]. Furthermore, ACA can be used in fine pitch applications as small as 40-um pad width and 80-um pad pitch. [26], [27]. Static and cyclic bending tests performed in [27] also indicate that the ACA joints are featured with sufficient reliability for consumer electronics.

In this work, the ACA from DELO is used to bond the SoC onto the interposer. Silver particles with a diameter of 1 3 um are dispersed in the adhesive. A cross-section view of the overall flip chip assembly process is shown in Fig. 6. In the initial stage, the adhesive is not conductive in any direction, as conductive particles do not touch each other due to their tiny particle size and low density dispensed in adhesive, as shown in Fig. 6a. When pressure is applied, the conductive particles between the chip pad and substrate are deformed or breached, so the electrical contact can be made in vertical direction (perpendicular to the plane of the die), while in the horizontal direction it still keeps non-conducting, as shown in Fig. 6b. During the assembly process of this work, a thin layer of ACA is applied over the printed leads on the interposer (Fig. 7b).The flip chip is then aligned and attached onto the interposer using a fine placer LEICA-MZ75. During this packaging process, required pressure and heat are generated by the fine placer. The interposer with the attached SoC is shown in Fig. 7b. In order to ensure the electrical and mechanical contacts between the die and substrate, a protecting mold (medical compatible epoxy 


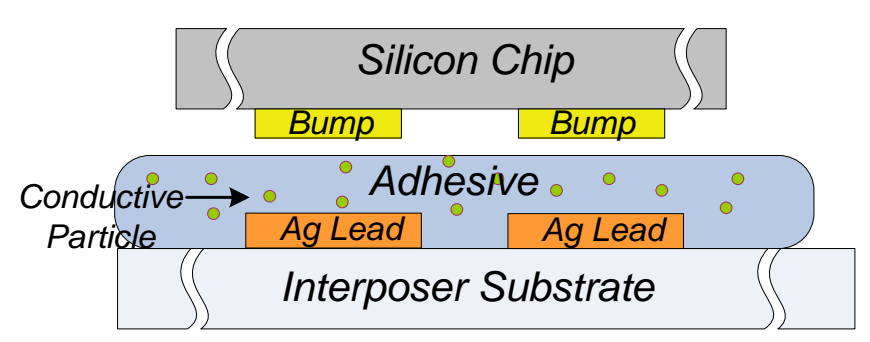

(a)

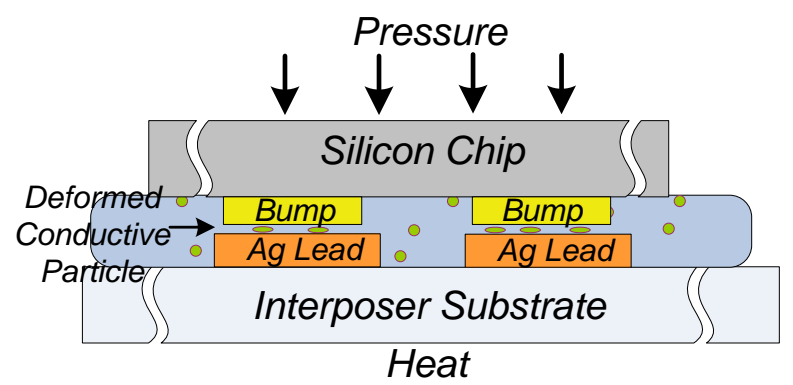

(b)

Fig. 6. Assembly process using ACA. (a) Silicon chip alignment. (b) Thermocompression bonding.

from EPOXY-Tech) is applied over the flip chip area, as shown in Fig. 7c. Finally, the interposer is flipped and attached to a larger PI mid-layer using ACA to form a sensor node, where several passive components are applied to configure the chip to a proper working condition, as shown in Fig. 7d. The sensor node is stand-alone with Active Cable interfaces printed on its four edges. It can be easily used for fast test or quick Bio-Patch assembly. Figure 8 shows the final Bio-Patch assembly which is accomplished by using conductive paste to mount the sensor node on the printed circuit board. The conductive electrode is formed by a thin silver plate on the PI substrate, where silver ink is inkjet printed on the PI substrate to form the electrode pattern and sintered in an oven at $145{ }^{\circ} \mathrm{C}$ for 1 hour. The fabricated silver electrode has a diameter of $15 \mathrm{~mm}$, and it is attached

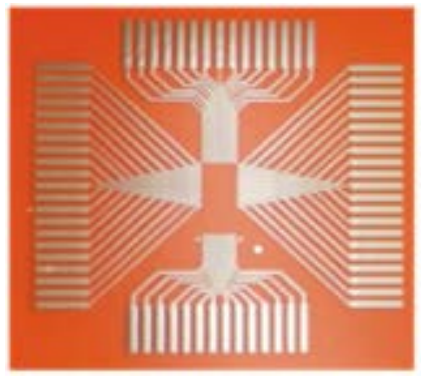

(a)

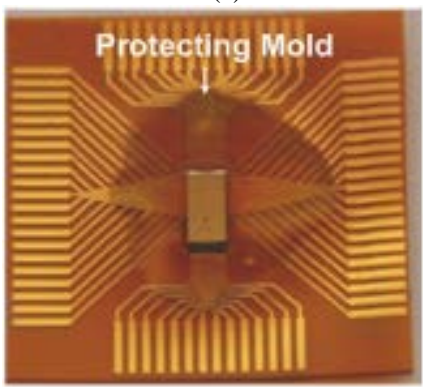

(c)

Fig. 7. SoC bonding and packaging. (a)Interposer on PI substrate. (b) Flip chip bonding with ACA. (c) Protecting mold. (d) Sensor node assembly.

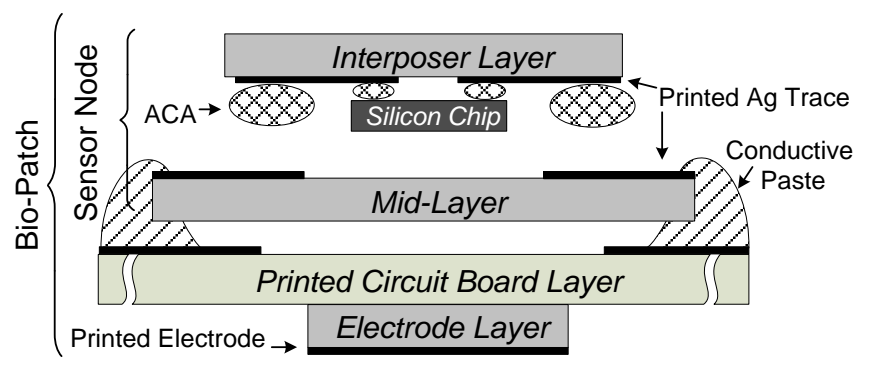

Fig. 8. Bio-Patch final assembly on PI substrate.

beneath the Bio-Patch. The electric connection between the electrode and a sensor node (one input of the AFE) is made via through-hole on the substrate. The performance evaluation of the printed electrodes for ECG measurement (including contact impedance variation over frequencies, optimal electrodes size and distance, and bending tests) has been reported in [28]. From the measurement results, it can be observed that the printed electrodes offer a comparable performance with the commercial pre-gelled electrodes.

The final assembled Bio-Patch prototype is shown in Fig. 9, with the size of $16 \mathrm{~cm} \times 16 \mathrm{~cm}$. The use of PI substrate and the integration of the bare die on the PI enable an ultra-thin patch solution with a total thickness less than $1.5 \mathrm{~mm}$. 8 SNs are deployed and mounted on specific points of a printed flexible circuit board layer (corresponding to 8 positions on the subject's chest), and the $\mathrm{MN}$ is located in the middle of the patch. A flat soft battery from Enfucell $(0.7 \mathrm{~mm}$ thickness, $1.4 \mathrm{~g}$ weight) is employed as the power supply. As shown in Fig. 9, the face-up battery is for demonstration purpose, the one facing downward is the actually working battery. Owning to its ultra-low power design, the power consumption is $15 \mu \mathrm{W}$ for a $\mathrm{MN}$, and $20 \mu \mathrm{W}$ for a SN. The total power consumption of the proposed Bio-Patch (1 MN plus $8 \mathrm{SNs}$ ) is only $175 \mu \mathrm{W}$. In this case, the Bio-Patch (excluding the wireless module) has a battery life of around 7 days with a single $3.0 \mathrm{~V} 10 \mathrm{mAh}$ Enfucell soft battery. Since wireless transmission module is recognized as a power-hungry component, it dominates the lifetime for the battery-operated wireless sensing devices. By using high-capacity lithium coin battery and low power wireless communication link (e.g. Bluetooth Low Energy), the proposed

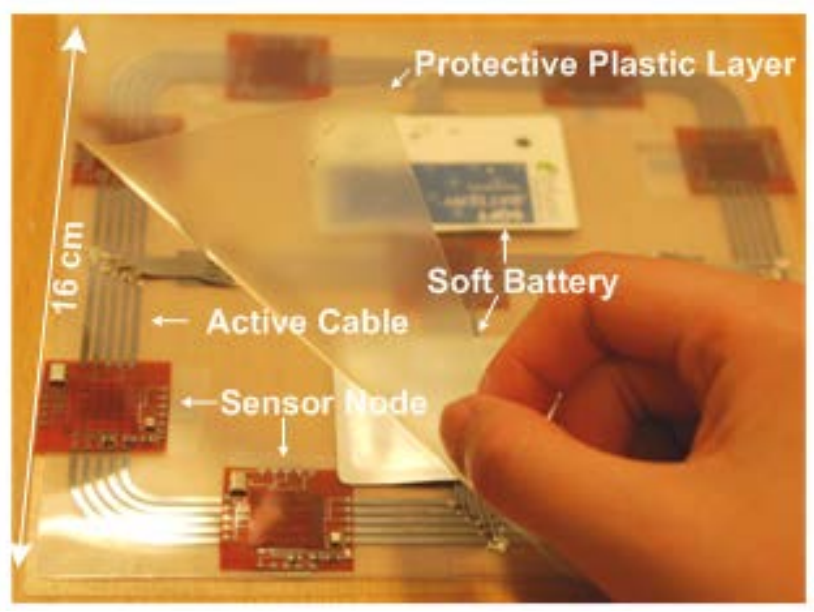

Fig. 9. Bio-Patch assembly. 


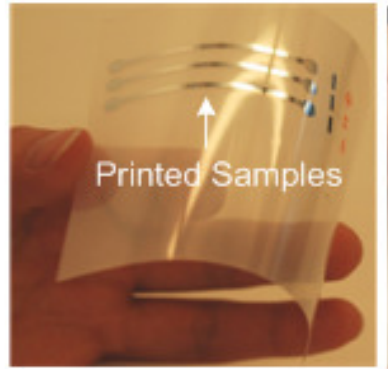

(a)

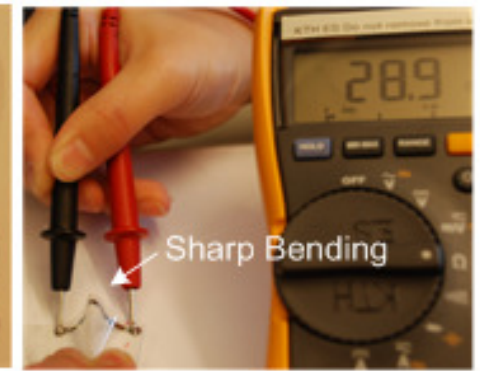

(b)
Fig. 10. Static bending test. (a) Test samples printed on PI. (b) Sharp folding.

Bio-Patch is capable of operating around 7 days for continuous monitoring. To protect the circuit, a thin plastic foil is applied to cover the patch. The Bio-Patch can be easily attached to a subject's chest by an elastic bandage or utilizing medical compatible adhesive at the bottom layer to ensure a good contact.

\section{B. Reliability Test}

Static bending test is performed to assess the reliability of the printed sliver traces on the PI substrate. 3 identical samples are printed on PI with a length of $4.5 \mathrm{~cm}$ and width of $2 \mathrm{~mm}$, as shown in Fig. 10a. During tests, a multimeter is used to measure the DC resistance of the printed silver trace. We gradually decrease the bending radius of the printed samples to examine if metal crack or peel-off can occur under any condition. Throughout the entire experiment, no metal crack or peel-off occurs, the samples keep an almost consistent conductivity even when the radius is decreased to $4 \mathrm{~mm}$ (which means the sample is spiral-wound of 4 turns, a quite large bending comparing with the sample size). Finally, we make an extreme bending test by folding the sample hard to form a sharp edge, as shown in Fig $10 \mathrm{~b}$. From the figure, it can be observed that the connection of the silver trace keeps intact with the resistance of around $29 \Omega$. The measurement results prove that the printed Active Cable can offer a reliable interconnection even in the case of extreme folding.

A dynamic bending analysis of the inkjet-printed silver conductors on a polymer substrate is recently reported in [29], where the reliability and durability issues of the printed metal traces on flexible substrates are investigated. A 'bending machine' is applied in this dynamic bending test. The measurement results show that inkjet-printed traces prove to have good tolerance against bending, in addition, the durability and reliability of the inkjet printed silver traces are much better than their counterpart: etched copper samples [29].

\section{Physiological Recordings}

Initial measurement is made by applying the Bio-Patch prototype on the chest with the help of an elastic bandage. The concurrent multi-channel body surface potential recordings are conducted after the system completes the sensor node scan process SNCS. The synchronous sampling command ('Syn-Sample') is broadcasted to trigger the circuit embedded inside each SN to work simultaneously. This method ensures
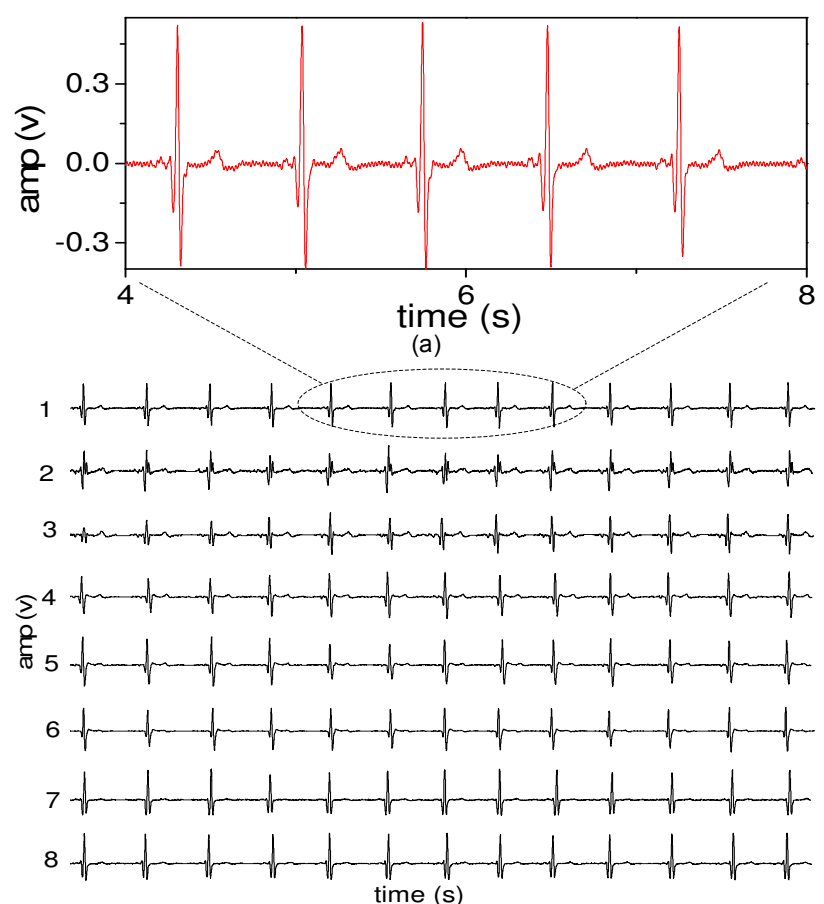

(b)

Fig. 11. Obtained concurrent ECG signals. (a) ECG segment from SN-1. (b) Concurrent ECG from chest positions 1-8.

that the data sampled at each chest point are recorded at the same time instant. The front end read-out circuit located in each SN has the same configuration: with a gain of $60 \mathrm{~dB}$ and a 3-dB bandwidth of $0.3 \mathrm{~Hz} \sim 1 \mathrm{kHz}$. Fig. $11 \mathrm{~b}$ shows the collected concurrent ECG data from SN positions 1-8, with a total time period of 12 seconds. It can be observed that the amplitude of the recorded ECG signal varies due to different sensing positions. To further verify the signal quality, a segment of ECG signal from $\mathrm{SN}-1$ is enlarged for illustration in Fig. 11a. It can be observed that a clear ECG waveform is obtained. Since all the experiments are conducted in a typical electric laboratory, a slight $50-\mathrm{Hz}$ power line noise can be observed.

For the long-term and homecare monitoring environment, the proposed Bio-Patch faces several challenges which may include: signal degradation resulting from the electrode damage due to the long-time skin-electrode contact; and the motion artifact which is generated from users' daily activities. Non-contact bioelectric signal measurement is an efficient approach [30]. With this method, the bioelectric signals (including the ECG signal) can be measured through the clothes/textile without direct skin-electrode contact, thus it can get rid of skin irritation and signal degradation problems. Also, the methods introduced in [31] and [32] can be applied in the Bio-Patch design to suppress the motion noise.

\section{CONCLUSION}

This paper presents a wearable hybrid Bio-Patch prototype utilizing System-on-Chip and high resolution inkjet printing technology for concurrent body surface potential measurement. 
The integrated Bio-Electric SoC is featured with low power and low noise. It achieves $90 \mathrm{~dB}$ CMRR and $56 \mathrm{nV} / \sqrt{\mathrm{Hz}} \mathrm{INR}$ density. The SoC fulfills the functional requirements of bio-potential sensing system, including signal sensing and processing, digitizing, data buffer and transmission. The tunable gain and programmable bandwidth enable the Bio-Patch to accommodate a wide range of bio-electric signal, and allow great freedom of choosing electrodes type, size and distance.

Instead of traditional standard package, an inkjet printed interposer on PI substrate is used as the silicon carrier. The distinct advantages of this approach include reduced package size, smaller thickness and more flexible to fit the body convexity. User comfort is improved by eliminating the cumbersome connecting cables with the sensor nodes serial connection method.The reliability of inkjet printed silver traces is evaluated through the bending test. The results indicate that the printed silver traces on PI substrate can be reliably employed in the fabrication of conductive electrodes, Active Cable and printed flexible circuit board. The system reliability is further enhanced by Active Cable's redundant architecture which can effectively reduce the risk of connection failure between sensor nodes. To evaluate the performance of the PI-base inkjet-printed Bio-Patch prototype, preliminary in-vivo test is applied. High quality concurrent ECG signals are successfully measured from 8 chest points. It is worth noting that up to $14 \mathrm{SNs}$ are supported by the system. Additional sensor nodes may also be added when increased spatial resolution is needed. The proposed system is used for body surface potential measurement, yet it could also be adapted and applied for other wearable healthcare applications.

\section{ACKNOWLEDGMENT}

The authors would like to thank Dr. Fredrik Jonsson for his comments on Bio-Patch test.

\section{REFERENCES}

[1] H. Zhang, Y.J. Qin, and Z.L Hong, "A 1.8-V 770-nW biopotential acquisition system for portable applications,” IEEE Biomedical Circuits and Systems Conference (BioCAS 2009), pp.93-96, 2009.

[2] B.D. Farnsworth, D.M. Talyor, R.J.Triolo, and D.J.Young, "Wireless in vivo EMG sensor for intelligent prosthetic control," Int. Solid-State Sensors, Actuators and Microsystems Conference (TRANSDUCERS 2009). pp.358-361, 2009.

[3] Y.T. Zhang, Y.S. Yan, C.C.Y. Poon, "Some Perspectives on Affordable Healthcare Systems in China," IEEE 29th Annual International Conference of the Engineering in Medicine and Biology Society, (EMBS 2007) pp.6154, Aug. 2007.

[4] A. Casson, D. Yates, S. Smith, J. Duncan, and E. Rodriguez-Villegas, "Wearable Electroencephalography," IEEE Engineering in Medicine and Biology Magazine, vol.29, no.3, pp.44-56, May 2010.

[5] "Heart Disease and Stroke Statistics 2010 Updates: A Report,” American Heart Association Statistics Committee and Stroke Statistics Subcommittee. [Online].

[6] H. Koch, A. Richter, R. Kursten, and M. Zabel, "Composition of approximated body-surface-potential-maps by utilizing a common 12-lead-ECG device," IEEE Transactions on Biomedical Engineering, vol.52, pp.463-470, Mar. 2005.

[7] H.C. Powell, M.A. Hanson, and J. Lach, "On-Body Inertial Sensing and Signal Processing for Clinical Assessment of Tremor," IEEE Transactions on Biomedical Circuits and Systems, vol.3, no.2, pp.108-116, April 2009.
[8] C.-T. Lin, C.-J. Chang, B.-S. Lin, S.-H. Hung, C.-F. Chao, and I-J. Wang, "A Real-Time Wireless Brain-Computer Interface System for Drowsiness Detection," IEEE Transactions on Biomedical Circuits and Systems, vol.4, no.4, pp.214-222, Aug. 2010.

[9] E. Halonen, A. Halme, T. Karinsalo, P. Iso-Ketola, M. Mäntysalo, and R. Mäkinen, "Dynamic Bending Test Analysis of Inkjet-Printed Conductors on Flexible Substrates," Electronic Components and Technology Conference (ECTC2012), 2012.

[10] M. Singh, "Inkjet Printing_Process and Its Applications," Adv. Mater., vol. 22: pp. 673-685. Feb. 2010.

[11] P. Kunnari, E. Laine, K. Niittynen and J. Koskinen, "Utilizing inkjet printing to fabricate electrical interconnections in a System-in-Package," ELSEVIER Microelectronic Engineering, Vol. 87, Issue 11, pp. 2382-2390, 2010.

[12] K. Saarinen, L. Frisk, and L. Ukkonen, "Effects of Cycling Humidity on the Performance of RFID Tags With ACA Joints," IEEE Transactions on Reliability,vol.61, pp.23-31, Mar. 2012.

[13] M. Mäntysalo, L. Xie, et al "System Integration of Smart Packages Using Printed Electronics," Electronic Components and Technology Conference (ECTC2012), 2012.

[14] J. Chen, L. Rong, F. Jonsson, G. Yang, and L.-R. Zheng, “The Design of All-Digital Polar Transmitter based on ADPLL and Phase Synchronized $\Delta \Sigma$ Modulator," IEEE Journal of Solid-State Circuits (JSSC) vol.47, no.5, pp.1154-1164, May 2012.

[15] G. Yang, J. Chen, Y. Cao, T. Hannu, and L-R. Zheng, “A novel wearable ECG monitoring system based on Active-Cable and Intelligent Electrodes," IEEE Proc of 10th Int. Conf. on e-Health Networking, Applications and Services (HealthCom2008), pp.156-159, Jul. 2008.

[16] Q. Wan, G. Yang, Q. Chen, and L- R. Zheng, "Electrical Performance of Inkjet Printed Flexible Cable for ECG Monitoring," 20th Electrical Performance of Electronic Packaging and Systems (EPEPS 2011), 2011.

[17] R. F. Yazicioglu, P. Merken, R. Puers, and C. V. Hoof, "A 200 uW Eight-channel EEG acquisition SoC for Ambulatory EEG Systems," IEEE Journal of Solid-State Circuits (JSSC), vol. 43, no.12, pp. 3025-3028, Dec. 2008.

[18] G. Yang, J. Chen, F. Jonsson, H. Tenhunen, and L-R. Zheng, "A Multi-Parameter Bio-Electric SoC Sensor with Integrated 2-Wire Data Transmission Protocol for Wearable Healthcare System," Design, Automation \& Test in Europe Conference \& Exhibition(DATE 2012), pp.443-448, Mar. 2012.

[19] G. Yang, J. Chen, F. Jonsson, T. Hannu, and L-R. Zheng, "A 1.0 V 78 uW reconfigurable SoC embedded in an Intelligent Electrode for continuous ECG applications," IEEE 31st Annu. Int. Conf. of the Engineering in Medicine and Biology Society(EMBC2009), pp.2316-2319, Sep. 2009.

[20] G. Yang, J. Chen, T. Hannu, and L-R. Zheng, "An SoC solution for Intelligent Electrodes and Active-cable used in a Wearable ECG monitoring system," Int. Conf. on Biomedical Electronics and Devices (BIODEVICES 2009), pp.209-213, Jan. 2009.

[21] Y. Feng, L. Xie, et al "Fabrication and Performance Evalutation of Ultralow-cost Inkjet-printed Chipless RFID Tags," Large-area, Organic \& Printed Electronics Conventions (LOPE-C 2012) 2012 (in press).

[22] B. An, X. Cai, H. Chu, X. Lai, F. Wu, and Y. Wu, "Flex reliability of RFID inlays assembled by anisotropic conductive adhesive," in Proceedings of HDP'07, pp. 60-63, 2007.

[23] X. Cai, B. An, Y. Wu, F. Wu, and X. Lai, "Research on the contact resistance and reliability of flexible RFID tag inlays packaged by anisotropic conductive paste," 9th International Conference on Electronic Packaging Technology \& High Density Packaging (ICEPT-HDP'08), pp. 129-133, Jul. 2008.

[24] J. S. Rasul, "Chip on paper technology utilizing anisotropically conductive adhesive for smart label applications," Microelectronics Reliability, vol. 44, Issue 1, pp. 135-140, Jan. 2004.

[25] Y. Li and C. P. Wong, "Recent advances of conductive adhesives as a lead-free alternative in electronic packaging: Materials, processing, reliability and applications,” Elsevier, Materials Science and Engineering, vol. 51, no. 1-3, pp.1-35, 2006.

[26] R. R. Tummala, E. J. Rymanszewski, and A. G. Klopfenstein, MicroelectronicsPackaging Handbook—Subsystem Packaging, Part III, $2^{\text {nd }}$ ed., p. 628, United States of America: Chapman \& Hall, 1997.

[27] S.-T. Lu, and W.-H. Chen, "Reliability and Flexibility of Ultra-Thin Chip-on-Flex (UTCOF) Interconnects With Anisotropic Conductive 
Adhesive (ACA) Joints,” IEEE Transactions on Advanced Packaging, vol.33, pp.702-712, Aug. 2010.

[28] L. Xie, G. Yang, M. Mäntysalo, L. L. Xu, F. Jonsson, and L.-R. Zheng "Heterogeneous Integration of Bio-Sensing System-on-Chip and Printed Electronics" IEEE Journal on Emerging and Selected Topics in Circuits and Systems(JETCAS), vol.2, no.4, pp.672-682, Dec. 2012.

[29] E. Halonen, A. Halme, T. Karinsalo, P. Iso-Ketola, M. Mantysalo, and R. Makinen, "Dynamic bending test analysis of inkjet-printed conductors on flexible substrates," IEEE 62nd Electronic Components and Technology Conference (ECTC), pp.80-85, May. 2012.

[30] Y.M. Chi, Tzyy-Ping Jung, and G. Cauwenberghs, "Dry-Contact and Noncontact Biopotential Electrodes: Methodological Review," IEEE Reviews in Biomedical Engineering, vol.3, pp.106-119, 2010.

[31] J.G. Webster, "Reducing Motion Artifacts and Interference in Biopotential Recording," IEEE Transactions on Biomedical Engineering, vol.BME-31, no.12, pp.823-826, Dec. 1984.

[32] Y.M. Chi, and G. Cauwenberghs, "Micropower non-contact EEG electrode with active common-mode noise suppression and input capacitance cancellation," IEEE 31st Annu. Int. Conf. of Engineering in Medicine and Biology Society (EMBC 2009), pp.4218-4221, Sept. 2009.

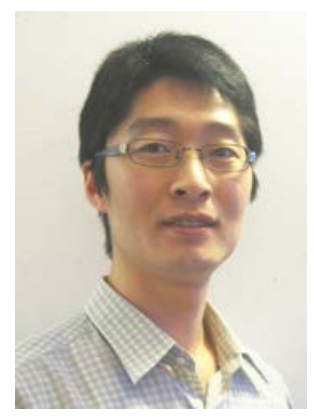

Geng Yang received his B.S. and M.S. degrees from the College of Biomedical Engineering and Instrument Science, Zhejiang University, Hangzhou, China, in 2003 and 2006 respectively. He is currently working toward the Ph.D. degree in iPack Vinnova Excellence center, the school of Information and Communication Technology (ICT), Royal Institute of Technology (KTH), Stockholm, Sweden. $\mathrm{He}$ developed low power, low noise bio-electric SoC sensors for body sensor network. His research interests include mixed-mode IC design for wearable bio-devices, intelligent sensors, and low-power biomedical microsystem.

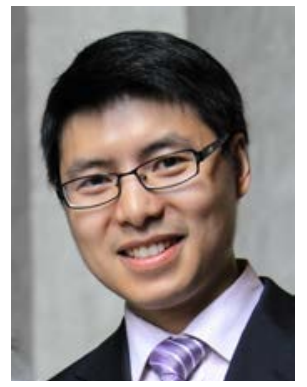

Jian Chen received the B.S. degree in electronic engineering and the M.S. degree in microelectronics from Shandong University, China, in 2003 and Fudan University, China, in 2006, respectively.

During 2007 - 2010, he conducted a joint research project between iPack Vinnova Excellence Center of Royal Institute of Technology (KTH) and Catena Wireless Electronics AB, Sweden, while working toward the Ph.D. degree. From 2011 until February 2012, he worked at Catena Wireless Electronics AB, Sweden as an RF Analog Circuit Designer. He is currently an RFIC Designer at Ericsson AB, Stockholm, Sweden. His research interests include mixed signal design and system level modeling for broadband radio frequency (RF) applications, particularly on phase noise optimization of RF oscillators, all-digital PLL (ADPLL), and digitally-intensive RF transmitter.

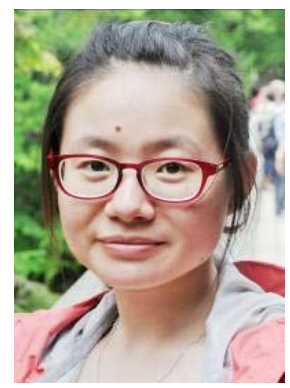

Li Xie received the B.Eng. degree in Electronic Information Engineering from Beihang University, China, in 2003, and the M.S. degree in Information and Communication from Beijing Institute of Technology, China, in 2009. She is currently working toward a Ph.D. degree in iPack Vinnova Excellence center, the school of Information and Communication Technology, Royal Institute of Technology (KTH), Stockholm, Sweden. Her main interests include printed electronics, flexible electronics, heterogeneous system integration and its application for wearable devices and intelligent packages.

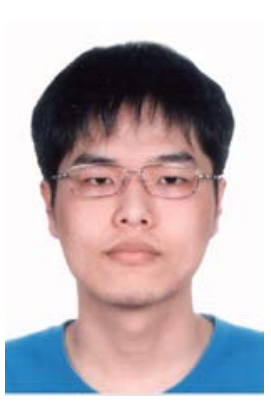

Jia Mao received the B.Eng. degree in 2006 from Southeast University, China and M.Sc. degree in 2009 from Royal Institute of Technology (KTH), Sweden, in Electrical Engineering. Since 2009, he has been a Research Engineer with Department of Electronic System, KTH, where he is currently working towards the Ph.D degree. His research interests include ultra low power analog and mix signal circuit design, UWB circuit and system and wireless sensor interface.

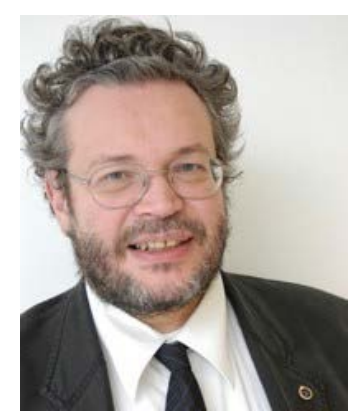

Hannu Tenhunen (S'83-M'90) received the Diploma Engineer degree in electrical engineering and computer sciences from Helsinki University of Technology, Helsinki, Finland, in 1982 and Ph.D. degree in microelectronics from Cornell University, Ithaca, NY, in 1986.

During 1978-1982, he was with Electron Physics Laboratory, Helsinki University of Technology. From 1983 to 1985, he was with Cornell University as a Fullbright Scholar. From September 1985, he was with the Signal Processing Laboratory, Tampere University of Technology, Tampere, Finland, as an Associate Professor. He was also a Coordinator of the National Microelectronics Program of Finland during 1987-1991. Since January 1992, he has been with the Royal Institute of Technology, Stockholm, Sweden, where he is a Chair Professor in electronic system design. His current research interests are very large scale integration (VLSI) circuits and systems for wireless and broadband communication, and related design methodologies and prototyping techniques. He has contributed significantly in microelectronics research and education in Europe. He has been actively involved in several EU programs on VLSI/system-on-a-chip.

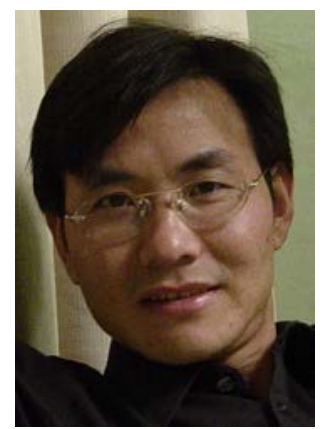

Li-Rong Zheng (M'01) received the Ph.D. degree in electronic system design from the Royal Institute of Technology (KTH), Stockholm, Sweden, in 2001.

He is a professor chair in media electronics at the Swedish Royal Institute of Technology (KTH), founder and director of iPack VINN Excellence Center, and Senior Specialist of Ericsson Networks in Stockholm, Sweden. He received his Ph. D. degree in electronic system design from the Royal Institute of Technology (KTH), Stockholm, Sweden in 2001. Since then, he was with KTH as a research fellow and project leader in Laboratory of Electronics and Computer Systems. He became an associate professor in electronics system design in 2003 and a full professor in media electronics at KTH in 2006. Dr. Zheng is a guest professor of the state key laboratory of ASICs and Systems at Fudan University in China since 2008, and a distinguished professor of Fudan University since 2010. His research experience and interest includes electronic circuits and systems for ambient intelligence and media applications, wireless SoC/SiP for sensing and identification, and signal integrity issues in electronic systems. He has authored and co-authored over 200 international reviewed publications, covering areas from electronic devices and thin film technologies, VLSI circuit and system design, to electronics systems and wireless sensors. 